\title{
Study on Purification of Chlorogenic Acid from Flos Lonicera Japonicae by Ionic Liquid Modified Macroporous Resin
}

\author{
Sun Yang ${ }^{1,}$, , Chen Hongyue ${ }^{1, b}$, Xu Chao ${ }^{1, ~ c}$, Song Ding ${ }^{1, ~ d}, \mathrm{Hu} \mathrm{Yi}^{1, \mathrm{e}}$ \\ ${ }^{1}$ School of Pharmaceutical Sciences of Nanjing Tech University, Nanjing, China. \\ axiaosunxiansen@njtech.edu.cn, b1028761352@qq.com, chyshyshine@foxmail.com, \\ d912209294@qq.com, ehuyi@njtech.edu.cn
}

\begin{abstract}
Keywords: Flos Lonicera Japonicae, Chlorogenic Acid, Ionic liquids, Macroporous resin, Purification
\end{abstract}

\begin{abstract}
Ionic liquids (ILs) were used to modify macroporous resins (MARs) to purify chlorogenic acid (CGA) with Flos Lonicera Japonicae (FLJ) extract as raw material. By comparing the static adsorption and desorption effects of four MARS, the NKA-2 resin was determined as the best purification material. The NKA-2 resin was modified by ILs to obtain the modified resin NKA-2-IL and its structure was characterized by infrared spectroscopy. The optimum conditions for the purification of NKA-2-IL MARS were investigated by single-factor experiments: sample concentration was $0.25 \mathrm{mg} / \mathrm{mL}$, eluent was $70 \%(\mathrm{v} / \mathrm{v})$ ethanol, solution $\mathrm{pH}$ was 3.0 , sample speed was $0.4 \mathrm{~mL} / \mathrm{min}$, elution rate was $0.6 \mathrm{~mL} / \mathrm{min}$ and aspect ratio was 30: 1 . Under this condition, the purity of CGA increased from $5.13 \%$ to $69.08 \%$, CGA yield was $89.5 \%$ and the purity of isochlorogenic acid (ICGA) was $22.12 \%$. The results showed that the purification of CGA by NKA2-IL MARS was significantly improved compared with that reported in the literature.
\end{abstract}

\section{Introduction}

FLJ is one of the commonly used Chinese herbal medicines and it has the functions of detoxification, cool wind cooling, liver protection and gallbladder, etc. FLJ has such an effect because it contains the active ingredient CGA playing a major role. CGA exists in the form of concentrate in the market and the price of CGA was different greatly on purity. A series of methods for the purification of CGA had been reported in the literature [1-2]. Based on the previous experiments, we have screened several different MARs and modified the resins with good preliminary purification effect by ILs, and then conditions of the purification process were optimized. The experiment applied FLJ extract as raw material and graft ILs on MARS to improve its separation and purification effect. The factors affecting the purifying effect of CGA on the purification process were investigated.

\section{Materials and methods}

Materials: FLJ was obtained from Xinyi Honeysuckle Agricultural Development Co., Ltd. All MARS were purchased from Shanghai Yuan ye Biotechnology Co., Ltd and all Reagent were obtained from Sinopharm Group Chemical Reagent Co., Ltd.

Analytical method: Analyzing experimental samples with a Dai an U3000 HPLC. The gradient elution was performed using methanol-water as the mobile phase at a wavelength of $327 \mathrm{~nm}$. The standard formula for CGA yield was $y=0.999 x+1.296\left(r^{2}=0.999, x\right.$, concentration, $\mathrm{g} / \mathrm{ml}$; $\mathrm{y}$, the peak area).

Preparation of $\boldsymbol{F L J}$ extract: A certain amount of pectinase was weighed to prepare an aqueous solution of pectinase at a concentration of $0-0.7 \mathrm{mg} / \mathrm{mL}$ and the $\mathrm{pH}$ was adjusted in the range of 2.06.0. $2.0 \mathrm{~g}$ dried $F L J$ powder was placed in an aqueous pectinase solution and then placed in sonicator at $40^{\circ} \mathrm{C}$ for 30 minutes to 4 hours. After the end of reaction, the extract was filtered and the filtrate was concentrated to $15 \mathrm{~mL}$ and $1 \mathrm{~mL}$ of the filtrate was diluted 625 times. The diluted solution was injected through a $0.45 \mu \mathrm{m}$ filter into a brown vial for subsequent HPLC analysis. 
Static adsorption and desorption of MARS: A suitable amount of MARS was put on a filter paper to remove water. $1.0 \mathrm{~g}$ MARS was weighed accurately into a $100 \mathrm{~mL}$ conical flask and added $30 \mathrm{~mL}$ $F L J$ extract at a mass concentration of $1.0 \mathrm{mg} / \mathrm{mL}$ in a bath shaker for $24 \mathrm{~h}$ and samples were taken at regular intervals to determine the concentration of CGA in the sample. By calculating the relationship between time and CGA adsorption, the kinetic curve of adsorption was obtained. The calculation formula was $Q=\frac{(C 0-C 1) V 0}{M}, A(\%)=\frac{C 0-C 1}{C 0} \times 100 \%$. In the formula, Q was the adsorption amount of CGA, mg/g; $\mathrm{C}_{0}$ was the initial mass concentration of the adsorption liquid, $\mathrm{mg} / \mathrm{mL}$; $\mathrm{C}_{1}$ was the mass concentration of the solution after adsorption, $\mathrm{mg} / \mathrm{mL} ; \mathrm{V}_{0}$ was the volume of the adsorption liquid, $\mathrm{mL}$; $\mathrm{M}$ was the MARS mass, $\mathrm{g}$.

The mentioned adsorbed resin was filtered to remove the extract. The filter paper was sucked to dry the surface moisture and the resin was placed in a $100 \mathrm{~mL}$ flask. $30 \mathrm{~mL} \mathrm{70 \%} \mathrm{(v/v)} \mathrm{ethanol} \mathrm{solution}$ was added and shaken in a $25^{\circ} \mathrm{C}$ bath shaker at $150 \mathrm{r} / \mathrm{min}$ for $5 \mathrm{~h}$, the desorption liquid mass concentration was determined and the desorption rate of the resin was calculated. The formula was D $(\%)=\frac{C 2 V 2}{(C 0-C 1) V 1} \times 100 \%$. In the formula, $\mathrm{V}_{1}$ was the desorption volume, $\mathrm{mL} ; \mathrm{C}_{2}$ was the desorption mass concentration, $\mathrm{mg} / \mathrm{mL} ; \mathrm{C}_{0}$ was the initial mass concentration of the adsorption liquid, $\mathrm{mg} / \mathrm{mL}$; $\mathrm{C}_{1}$ was the mass concentration of the solution after adsorption, $\mathrm{mg} / \mathrm{mL}$.

Chloromethylation Modification of NKA-2 Type Resin [3-4]: (1) $31 \mathrm{~mL}$ formaldehyde solution and $50 \mathrm{~mL}$ of 1, 4-butanediol were measured accurately in a three-necked flask and all of them was put into the three-necked flask on an ice-water bath. Then $33 \mathrm{~mL}$ of phosphorus trichloride were added slowly at a rate of $0.5 \mathrm{~mL} / \mathrm{s}$. At the end of the reaction, the solution was transferred to a separating funnel and the aqueous layer was discarded. The remaining oil layer was dried by adding an appropriate amount of anhydrous sodium sulfate, and then the intermediate reactant $\mathrm{ClCH}_{2} \mathrm{O}\left(\mathrm{CH}_{2}\right)_{4} \mathrm{ClCH}_{2} \mathrm{O}$ was obtained by distillation under reduced pressure.

(2) $20.0 \mathrm{~g}$ dried NKA-2 resin, $75 \mathrm{~mL} \mathrm{ClCH} 2 \mathrm{O}\left(\mathrm{CH}_{2}\right)_{4} \mathrm{ClCH}_{2} \mathrm{O}$ and $250 \mathrm{~mL}$ carbon tetrachloride were put into a three-necked flask for $12 \mathrm{~h}$. After the end of reaction, Sodium and zinc chloride were put into three-necked flask and the reaction system was transferred to a reflux apparatus at $50^{\circ} \mathrm{C}$ for $10 \mathrm{~h}$. After the end of reaction, the product was washed with deionized water and ethanol to obtain a chloromethylated NKA-2 modified resin.

Ionic Liquid Modification of NKA-2-CH2 $\mathbf{C l}$ Resin: In a $250 \mathrm{~mL}$ round bottom flask, $10.0 \mathrm{~g}$ NKA2-Cl resin and $20.0 \mathrm{~g}$ butyl imidazole and $100 \mathrm{~mL}$ chloroform were mixed to react at $70^{\circ} \mathrm{C}$ for $24 \mathrm{~h}$. After the reaction was over, the filtered product was washed with diethyl ether and dried in an oven at $40^{\circ} \mathrm{C}$. The target product namely butyl imidazole IL modified resin (NKA-2-IL) was obtained [5].

Optimization of the purification process of NKA-2-IL resin: Based on literature investigations and experimental explorations, we examined five important factors including the effect of $\mathrm{pH}$ on the adsorption characteristics, the effect of ethanol concentration on the desorption characteristics, the influence of the sample rate on the adsorption process, the effect of the aspect ratio on the adsorption process and the effect of the speed on the desorption process.

\section{Results and discussion}

Different types of resin screening: Table 1 showed the adsorption performance of CGA by four different types MARS. The adsorption and adsorption rates of CGA by the resin showed that NKA9, ADS-21, NKA-2 type resins all had a certain polarity. Polar CGA molecules and hydrogen bonds between the resin were easy to form a strong bond and it also showed that the polarity of the material and the resin was separated important factors affecting the adsorption performance [5-7]. The strongly polar NKA-9 resin showed a high adsorption rate. The non-polar D1300 resin had weaker interaction with CGA, which resulted in lower adsorption. The adsorption of NKA-2 resin was slightly lower than that of NKA-9 resin, but the desorption rate was the highest. Therefore, NKA-2 was selected as the most suitable MAR. 
Table 1 Study on static adsorption and desorption performance of MAR for CGA

\begin{tabular}{cccc}
\hline Resin type & Adsorption capacity $(\mathrm{mg} / \mathrm{g})$ & Adsorption rate $(\%)$ & Desorption rate $(\%)$ \\
\hline NKA-9 & 25.51 & 85.6 & 85.7 \\
ADS-21 & 23.34 & 77.8 & 89.9 \\
NKA-2 & 25.23 & 84.3 & 90.2 \\
D1300 & 20.46 & 70.1 & 81.9 \\
\hline
\end{tabular}

Infrared spectral characterization: Fig. 1 showed the infrared spectra of NKA-2 and NKA-2-IL type polystyrene resins. It was two absorption peaks around $3090 \mathrm{~cm}^{-1}$ and $3010 \mathrm{~cm}^{-1}$, which belong to the stretching vibration absorption peak of the $\mathrm{C}-\mathrm{H}$ bond on the benzene ring and the absorption peak at $1585 \mathrm{~cm}^{-1}$ is the benzene ring. The vibration absorption peaks of the skeleton showed obvious $-\mathrm{CH}_{2}$ stretching vibration peaks at $2963 \mathrm{~cm}^{-1}$ and $2926 \mathrm{~cm}^{-1}$ [8]. NKA-2-IL resin was a C-H stretching vibration peak of polystyrene resin at $2963 \mathrm{~cm}^{-1} ; 861 \mathrm{~cm}^{-1}$ was a N-H bending vibration and $925 \mathrm{~cm}^{-}$ ${ }^{1}$ was an inplane rocking vibration peak of $\mathrm{N}-\mathrm{H}$. The $\mathrm{C}=\mathrm{C}$ characteristic peak on the imidazole ring of the ionic liquid-modified group at $1150 \mathrm{~cm}^{-1}$ and the characteristic absorption peak of the $\mathrm{C}=\mathrm{N}$ group at $1565 \mathrm{~cm}^{-1}[5]$.

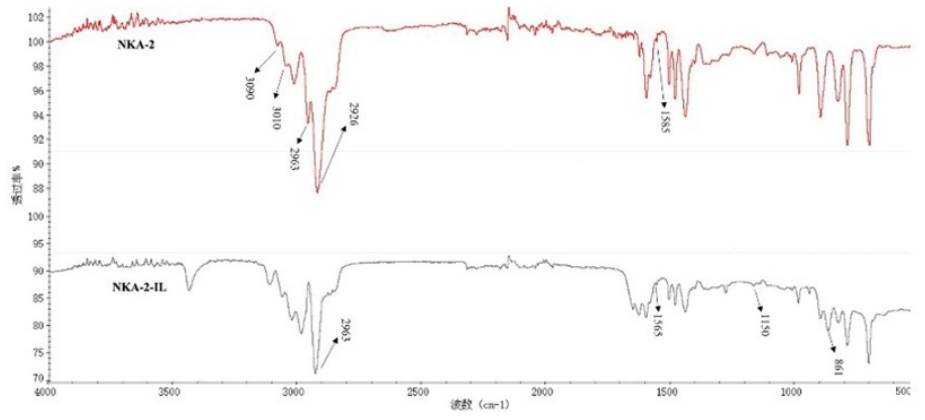

Fig. 1 Infrared Spectroscopic Characterization of NKA-2 and Modified NKA-2

Static Adsorption and Desorption of NKA-2-IL Resin: The test results of adsorption performance of CGA by NKA-2-IL resin were shown in Table 2.

Table 2 Static adsorption and desorption of chlorogenic acid by NKA-2-Cl resin

\begin{tabular}{cccc}
\hline Resin type & Adsorption capacity $(\mathrm{mg} / \mathrm{g})$ & Adsorption rate (\%) & $\begin{array}{c}\text { Desorption rate } \\
(\%)\end{array}$ \\
\hline NKA-2 & 25.23 & 84.3 & 90.2 \\
\hline NKA-2-IL & 26.81 & 86.6 & 94.1 \\
\hline
\end{tabular}

From Table 5, we found the adsorption of NKA-2-IL resin slightly increased, the adsorption rate increased and the desorption rate increased compared with NKA-2.

Optimization of Conditions of effect of pH on adsorption of NKA-2-IL resin: As shown in Fig.2, the adsorption of NKA-2-IL resin reached its maximum at $\mathrm{pH}$ 3.0. The reason may be the dissociation of CGA on ionic state under strong acid conditions, which resulted in a decrease on the adsorption amount [6]. With the increase of $\mathrm{pH}$, the degree of ionization of CGA also increase. The attractiveness between CGA and NKA-2-IL MARS gradually change into electrostatic effect and the adsorption capacity decreased resulting the adsorption capacity decreased. Therefore, $\mathrm{pH} 3.0$ was chosen as the initial $\mathrm{pH}$ of the sorption solution.

Optimization of Conditions of effect of ethanol concentration on the desorption rate of NKA-2IL resin: Ethanol was chosen as eluent because of the physicochemical properties of CGA and its own characteristics. From Fig.3, the desorption rate increased with the increase of ethanol concentration and reached peak at $70 \%(\mathrm{v} / \mathrm{v})$. When the ethanol concentration was higher than $70 \%$ $(\mathrm{v} / \mathrm{v})$, the desorption rate begun to decrease and indicated that the hydrophobic and hydrogen bonding existing between the modified NKA-2-IL resin and the CGA molecule had been destroyed. Ethanol concentration was too high resulting contrast of polarity between ethanol solution and CGA, which reduced CGA desorption rate [9]. Therefore, $70 \%(\mathrm{v} / \mathrm{v})$ concentration of ethanol was chosen as eluent. 
Optimization of Conditions of effect of loading rate on adsorption properties of NKA-2-IL resin: The NKA-2-IL resin was used for different loading speeds with $70 \%(\mathrm{v} / \mathrm{v})$ ethanol as eluent, $\mathrm{pH} 3.0$, elution rate $0.6 \mathrm{~mL} / \mathrm{min}$ and an aspect ratio 30:1. The dynamic adsorption performance was investigated. As shown in Fig. 4, CGA molecules had no enough time to be adsorbed on the inner surface of the NKA-2-IL type resin at a flow rate of $0.8 \mathrm{~mL} / \mathrm{min}$, which resulted in a decrease of adsorption effect. When the flow rate was $0.2 \mathrm{~mL} / \mathrm{min}$ and $0.4 \mathrm{~mL} / \mathrm{min}$, the difference

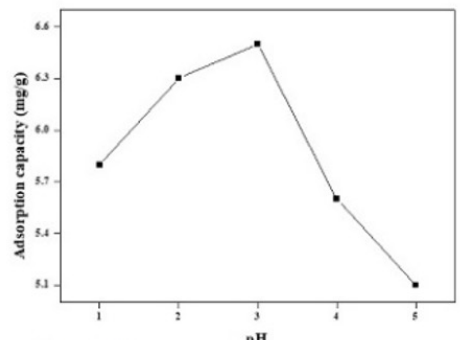

Fig.2 Effect of $\mathrm{pH}$ on adsorption

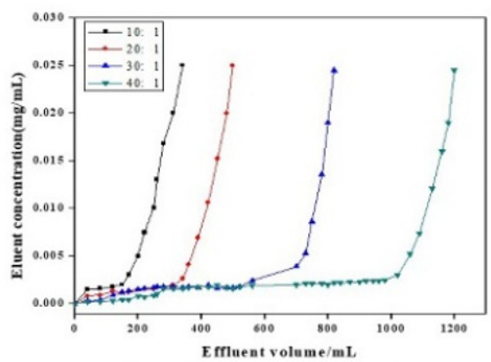

Fig. 5 Effect of aspect ratio on adsorption Properties of NKA-2-Cl resin

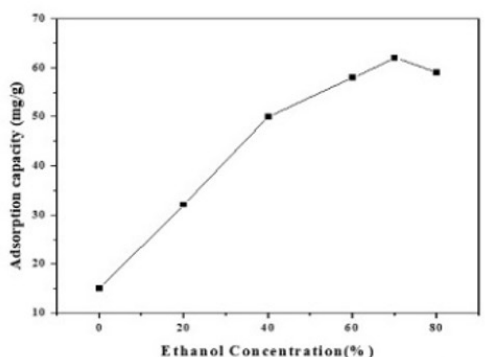

Fig.3 Effect of ethanol concentration and desorption rate

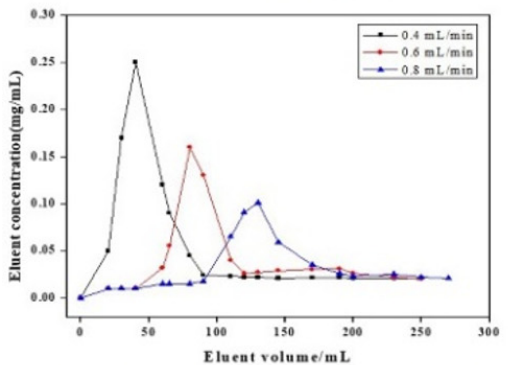

Fig.6 Effect of elution rate on desorption performance

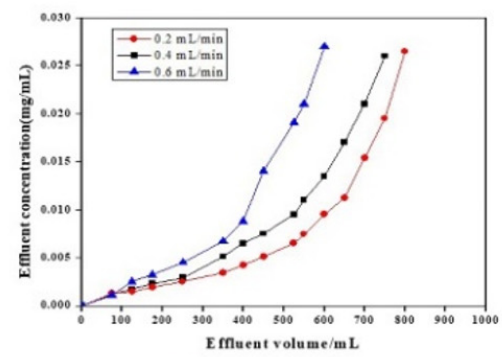

Fig.4 Effect of injection speed on adsorption performance

between the two leakage points was not large, but the treatment period of $0.2 \mathrm{~mL} / \mathrm{min}$ was longer than $0.4 \mathrm{~mL} / \mathrm{min}$. For a comprehensive comparison, $0.4 \mathrm{~mL} / \mathrm{min}$ was selected as the optimum sampling speed.

Optimization of Conditions of effect of aspect ratio on adsorption properties of modified NKA2-IL Resin: 70\% (v/v) ethanol was chosen as eluent and under the conditions of the aspect ratio 10: 1, pH 3.0, sample rate $0.4 \mathrm{~mL} / \mathrm{min}$ and an elution rate $0.6 \mathrm{~mL} / \mathrm{min}$, the dynamic adsorption properties of CGA were investigated at 20: 1, 30: 1 and 40: 1. Fig.5 showed that when the aspect ratio was 10: 1 and 20: 1, CGA was not adsorbed in time and the leakage phenomenon was more serious. As the aspect ratio increased, the residence time of the sample solution in the column changed longer, which was beneficial for the resin to adsorb CGA fully. When the aspect ratio was 30: 1 and 40: 1, the treatment volume was $800 \mathrm{~mL}$ and $1100 \mathrm{~mL}$ respectively. Considering actual production cost, 30: 1 was selected as NKA-2- IL resin dynamic adsorption of the aspect ratio.

Optimization of Conditions of effect of elution speed on desorption performance of modified NKA-2-IL Resin: The desorption performance at different elution rates was studied using a 70\% (v/v) ethanol solution as eluent at a condition of $\mathrm{pH} 3.0$, sample rate $0.4 \mathrm{~mL} / \mathrm{min}$ and aspect ratio 30: 1 . As shown in Fig.6, the elution peak was the most concentrated and there was no obvious tailing phenomenon when the elution rate was $0.6 \mathrm{~mL} / \mathrm{min}$. Therefore, $0.6 \mathrm{~mL} / \mathrm{min}$ was selected as the optimal elution rate.

\section{Summary}

NKA-2-IL MAR was used to separate and purify CGA from the crude extract of $F L J$. The optimal conditions were as follows: eluent was $70 \%(\mathrm{v} / \mathrm{v})$ ethanol, $\mathrm{pH} 3.0$, loading speed $0.4 \mathrm{~mL} / \mathrm{min}$, elution rate $0.6 \mathrm{~mL} / \mathrm{min}$, ratio of height to diameter $30: 1$. Under this condition, the purity of the CGA product reached $91.20 \%$. Among them, the purity of CGA was increased from $5.13 \%$ to $69.08 \%$, the yield 
was $89.5 \%$ and the purity of the ICGA was $22.12 \%$.

\section{Acknowledgements}

This work was financially supported by the National Natural Science Foundation of China (21676143), the Jiangsu Synergetic Innovation Center for Advanced Bio-Manufacture, Self-Owned Research Project from Key Laboratory of Material-Oriented Chemical Engineering (Grant No. ZK201603) and Qing Lan Project of Jiang Su Province.

\section{References}

[1] Z.H. Gong, G.P. Ren, Q.X, Shu and W.J. Xiao: J Instr Anal. Vol. 30 (2011), p. 85.

[2] Z.S. Saleh, R. Stanley and R. Wibisono: Int J Food Eng, Vol. 2 (2006), p. 88.

[3] Y.L. Shen, Y.F. Yang, B.J. Gao and G. Li: Chem J Chinese U, Vol. 28 (2007), p. 580.

[4] B. Gao, Q. Liu and L. Jiang: Chem Eng and Processing Process Intensification, Vol. 47 (2008), p. 852.

[5] C.J. Serpell, N.L. Kilah, P. J. Costa, V. Félix, and P.D. Beer: Angew Chem, Vol. 49 (2010), p. 5322.

[6] B.L. He and Z.Q. Shi: Polym Bull, Vol. 4 (2005), p. 13.

[7] Y.S. Wang and Y. Wang: China J of Chinese materia medica, Vol. 31 (2006), p. 961.

[8] Z.J. Tan, F. Li and X. Xu: Sep Purif Technol, Vol. 98, (2012), p. 150.

[9] B. Zhang, R. Yang, Y. Zhao and C. Z. Liu: J of Chromatography B Analytical Tech in the Biomedical and Life Sci, Vol. 867 (2008), p. 253. 